\title{
Engineering Thermo-pH Dual Responsive Hydrogel for Enhanced Tumor Accumulation, Penetration, and Chemo-Protein Combination Therapy
}

This article was published in the following Dove Press journal: International Journal of Nanomedicine

\section{Xiuping Pang ${ }^{1, *}$ \\ Shuang Liang ${ }^{1} * *$ \\ Tianqi Wang' \\ Shuangjiang $\mathrm{Yu}^{2}$ \\ Rui Yang' \\ Teng Hou' \\ Yongjun Liu' \\ Chaoliang $\mathrm{He}^{2}$ \\ $\mathrm{Na}$ Zhang'}

'Department of Pharmaceutics, Key Laboratory of Chemical Biology (Ministry of Education), School of Pharmaceutical Sciences, Shandong University, Jinan, Shandong Province 2500 I2, People's Republic of China; ${ }^{2}$ Key Laboratory of Polymer Ecomaterials, Changchun Institute of Applied Chemistry, Chinese Academy of Sciences, Changchun I30022, People's Republic of China

*These authors contributed equally to this work
Correspondence: Chaoliang $\mathrm{He} ; \mathrm{Na}$

Zhang

Email clhe@ciac.ac.cn;

zhangnancy9@sdu.edu.cn
Purpose: Combined chemotherapeutic drug and protein drug has been a widely employed strategy for tumor treatment. To realize both tumor accumulation and deep tumor penetration for drugs with different pharmacokinetics, we propose a structure-transformable, thermo-pH dual responsive co-delivery system to co-load granzyme B/docetaxel (GrB/DTX).

Methods: Thermo-sensitive hydrogels based on diblock copolymers (mPEG- $b$-PELG) were synthesized through ring opening polymerization. GrB/DTX mini micelles (GDM) was developed by co-loading these two drugs in $\mathrm{pH}$-sensitive mini micelles, and the GDMincorporated thermo-sensitive hydrogel (GDMH) was constructed. The thermo-induced gelation behavior of diblock copolymers and the physiochemical properties of GDMH were characterized. GDMH degradation and deep tumor penetration of released mini micelles were confirmed. The $\mathrm{pH}$-sensitive disassembly and lysosomal escape abilities of released mini micelles were evaluated. In vitro cytotoxicity was studied using MTT assays and the in vivo antitumor efficacy study was evaluated in B16-bearing C57BL/6 mice.

Results: GDMH was gelatinized at body temperature and can be degraded by proteinase to release mini micelles. The mini micelles incorporated in GDMH can achieve deep tumor penetration and escape from lysosomes to release GrB and DTX. MTT results showed that maximum synergistic antitumor efficacy of GrB and DTX was observed at mass ratio of $1: 100$. Our in vivo antitumor efficacy study showed that GDMH inhibited tumor growth in the subcutaneous tumor model and in the post-surgical recurrence model.

Conclusion: The smart-designed transformable GDMH can facilitate tumor accumulation, deep tumor penetration, and rapid drug release to achieve synergistic chemo-protein therapy. Keywords: structure-transformable, thermo-pH dual responsive, hydrogel, chemo-protein combination therapy

\section{Introduction}

Malignant tumors are a major public health problem worldwide. ${ }^{1-3}$ Protein drugs are one of the most potent biotherapeutics and have shown tremendous potential in cancer therapy. Protein drugs have the advantages of high therapeutic activity, high selectivity, and low toxicity to healthy cells. ${ }^{4,5}$ Many apoptotic proteins, including TRAIL, herceptin, and granzyme B (GrB), have been investigated for use in cancer therapy. ${ }^{6} \mathrm{GrB}$ is considered a direct "weapon", able to kill tumor cells in the immune system and has great potential to become a novel cytokine for tumor therapy. ${ }^{7,8} \mathrm{GrB}$ is a serine proteinase mainly secreted by immunologic effector cells including natural killer cells and cytotoxic T lymphocytes. GrB binds to target cells and is endocytosed with the help of perforin. ${ }^{9,10}$ 
The apoptosis of target cells is induced by internalized GrB mainly via caspase-dependent pathways. ${ }^{11-14}$ However, the therapeutic use of single protein drugs faces serious challenges including drug degradation, elimination following intravenous injection, poor tumor accumulation, low tumor penetration, and reduced antitumor efficacy. ${ }^{6,15,16}$ Many studies have been conducted on the combination of protein and chemotherapy drugs using drug delivery systems, which effectively deliver different drugs to the tumor site where they have synergistic antitumor efficacy. ${ }^{17-20}$ For example, the TRAIL and gambogic acid co-loaded folate-modified core/shell nanoparticulate complex, which exerts combined tumor therapy by regulating both intrinsic and extrinsic apoptotic pathways, was developed for cancer therapy. ${ }^{21}$ Additionally, Zhang developed cytokine interleukin-2 (IL-2) and doxorubicin co-loaded nanovesicles (NV-DOXIL-2) for cancer immunochemotherapy. After intravenous injection into melanoma-bearing mice, NV-DOXIL-2 accumulates in tumors and suppresses tumor growth. ${ }^{22}$ These studies were designed to deliver anticancer drugs to tumor sites through active or passive tumor targeting following systemic administration, and have shown satisfying antitumor effects. However, the systemic administration of $\mathrm{GrB}$, as a protein drug, has serious challenges including drug degradation and systemic toxicity. ${ }^{10,23-25}$ Therefore, it is necessary to choose a suitable drug-delivery system for GrB.

For GrB, peritumoral therapeutic platforms based on local delivery systems may provide a promising alternative to systemic administration. These advantages of peritumoral therapeutic platforms include precise administration, higher drug concentration at the tumor site, and significantly lower systemic toxicity. ${ }^{26-28}$ Hydrogel has attracted considerable attention as a potential local delivery system for cancer treatment. $^{29,30}$ In recent years, the design of temperaturesensitive hydrogels for controlled drug release has received considerable research attention because of its facile in situ gelation process at body temperature. ${ }^{31,32}$ For example, three kinds of drug (containing IL-2, IFN- $\gamma$, and doxorubicin)incorporating hydrogels were successfully prepared by Chen and coworkers, and showed synergistic anticancer efficacy through acting on different pathways in xenograft tumorbearing mice. ${ }^{33}$ Despite its potential, peritumoral therapy remains far from satisfactory. An important issue in this approach is the poor penetration of agents within tumors due to their dense tissues and high pressure. ${ }^{34}$ Accumulating data suggest that small-sized nanoparticles (less than $50 \mathrm{~nm}$ ) exhibit significant benefits for deep tumor penetration, but often suffer from poor tumor accumulation. ${ }^{35,36}$ Therefore, there is a need for a delivery system that combines the advantages of in situ-forming hydrogels and small-sized nanoparticles to achieve both tumor accumulation and deep tumor penetration.

Combining the advantages of hydrogels and small-sized nanoparticles, a novel structure-transformable thermo-pH responsive co-delivery system to deliver co-loaded chemoprotein drugs to the tumor site, transport drugs deep into the tumor tissue, and rapidly release drugs in tumor cells were successfully developed. Thermo-sensitive hydrogels based on diblock copolymers (mPEG- $b$-PELG) were synthesized using ring opening polymerization. GrB/DTX mini micelles (GDM) were developed by co-loading the two drugs in $\mathrm{pH}$-sensitive mini micelles, then GDM-incorporated thermo-sensitive hydrogel (GDMH) was constructed. The developed GDMH was expected to have the advantages of: (1) directly colocating different drugs within the tumor and diverting the drug from non-target organs to reduce toxicity and increase antitumor efficiency; (2) achieving deep tumor penetration due to the mini-sized nanoparticles; and (3) co-delivering GrB and DTX into the same tumor cells and rapidly releasing them to improve synergetic therapeutic effects (Figure 1). We believe that the developed smart-designed transformable GDMH can pave a new way for designing co-delivery system to facilitate tumor accumulation, deep tumor penetration, and rapid drug release to achieve synergistic chemo-protein therapy.

Herein, the pH-sensitive poly ( $\gamma$-glutamic acid)-poly (L-histidine) (PGA-PLH) was employed to prepare GDM. PGA is a negatively charged polyanion polymer in physiological environment. PLH possesses "proton pump effect" due to the side-chain imidazole groups, and its solubility could be affected by $\mathrm{pH}$ change (hydrophobic: uncharged, $\mathrm{pH} \sim 7.4$; hydrophilic: positive charge, $\mathrm{pH}<5.5)$. $\mathrm{GrB}$ and DTX would be co-loaded in the $\mathrm{pH}$-sensitive PGA-PLH mini micelles via electrostatic adsorption and hydrophobic interaction, respectively. Temperature-sensitive poly (ethylene glycol)-poly( $\gamma$-ethyl-L-glutamate) diblock copolymer (mPEG- $b$-PELG) was synthesized and applied to obtain transformable GDMH. The physical and chemical properties of mini micelles-incorporated hydrogel were characterized. The deep penetration capacity, lysosomal escape ability, codelivery efficiency, in vitro and in vivo antitumor efficacy were investigated, respectively. In addition, the preliminary safety evaluations were also performed.

\section{Materials and Methods}

\section{Materials}

DTX was purchased from Dalian Meilun Biotechnology Co., LTD. (Dalian, China). Cytochrome C (CC) $(\mathrm{Mw}=12,384)$ 
A

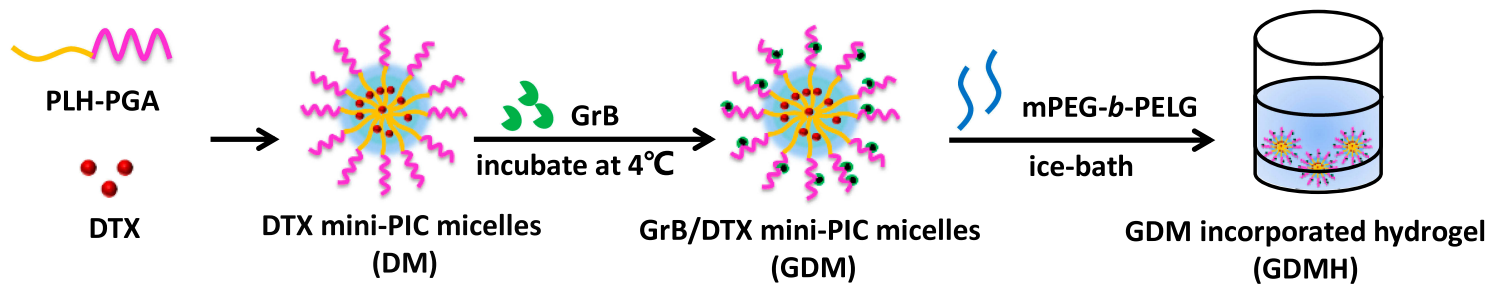

B

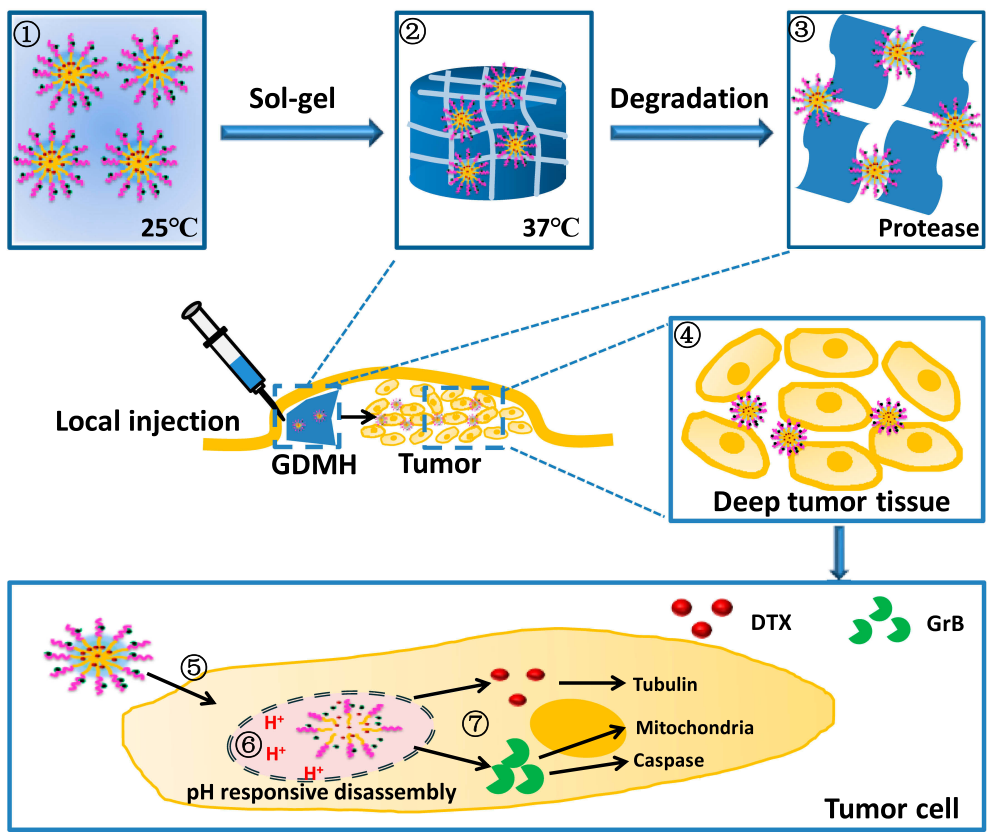

Figure I Schematic diagram of transformable injectable hydrogel as the local GrB/DTX co-delivery system for combination therapy. (A) The construction of structuretransformable hydrogel (GDMH). (B) The structure-transformable hydrogel could achieve sequentially responsive delivery: (1)-(3) The GrB/DTX pH-sensitive mini micelles (GDM) were incorporated into thermo-sensitive hydrogel (GDMH); (4) GDMH was degraded by proteinase and released mini micelles to achieve deep tumor penetration after be injected peritumorally; (5)-(7) Mini micelles disassembled and escaped from lysosomes via proton sponge effect to release GrB and DTX, then GrB and DTX played synergistic chemo-protein antitumor efficacy.

was purchased from Sigma-Aldrich (St. Louis, MO, USA). PGA-PLH (degrees of polymerization of PGA and PLH are 14 and 28, respectively) was synthesized by Nanjing Leon Biological Technology Co.,LTD. (Nanjing, China). Murine $\mathrm{GrB}(\mathrm{Mw}=28.9 \mathrm{kDa})$ was purchased from PeproTech, Inc. 3-(4,5-Dimethylthiazol-2-yl)-2,5-diphenyltetrazolium bromide (MTT) is the product of Solarbio (Shanghai, China). All other reagents were of analytical purity grade or higher, obtained commercially.

\section{Cell Cultures}

Murine melanoma cells (B16) were purchased from the Chinese Academy of Sciences (China). Cells were cultured in RPMI-1640 medium, supplemented with 10\% FBS, $1 \%$ penicillin and $1 \%$ streptomycin at $37{ }^{\circ} \mathrm{C}$ in an environment containing $5 \% \mathrm{CO}_{2}$.

\section{Animals}

Female C57BL/6 mice (weight: 20-22 g) were supplied by SPF (Beijing) Biotechnology Co.,Ltd. (Beijing, China). The animals were fed with a standard diet and allowed water ad libitum. All experiments were carried out in compliance with the Animal Management Rules of the Ministry of Health of the People's Republic of China and the Animal Experiment Ethics Review of Shandong University (Approval No. 18002).

\section{Synthesis of mPEG-b-PELG}

mPEG- $b$-PELG was synthesized according to our previous literature. ${ }^{37}$ As shown in Figure S1, mPEG-NH $\mathrm{NH}_{2}$ (3 g) was dissolved in toluene and residual water was removed by azeotropic distillation. Anhydrous N,N-dimethylformamide (DMF) and $\gamma$-ethyl-L-glutamate N-carboxyanhydride (ELG- 
NCA) ( $4.23 \mathrm{~g})$ were then added to the flask. The polymerization was performed at $30{ }^{\circ} \mathrm{C}$ for $72 \mathrm{~h}$ under $\mathrm{N}_{2}$ atmosphere. Then the solution was precipitated into cold diethyl ether. The reaction product was washed two times with diethyl ether and dried under reduced pressure.

\section{Preparation of Co-Loaded Mini Micelles-Incorporated Hydrogel}

DTX loaded mini micelles (DM) were prepared by the thin-film hydration method. In brief, PGA-PLH and DTX were dissolved in methanol and placed in a round-bottom flask. The solvent was removed by rotary evaporation at $40^{\circ} \mathrm{C}$ for 15 min to obtain a solid matrix. Residual methanol was removed under vacuum overnight at room temperature. The resultant thin film was hydrated with PBS $(\mathrm{pH}=7.4)$ at $40{ }^{\circ} \mathrm{C}$ for $10 \mathrm{~min}$ to obtain DM. Blank micelles were prepared using the same method, without the addition of DTX.

GrB was kept in an ice bath during the experiments to prevent degradation caused by temperature changes. GrB loaded mini micelles (GM) were prepared by adding $\mathrm{GrB}$ solutions to blank micelles and incubating for $24 \mathrm{~h}$ at $4{ }^{\circ} \mathrm{C}$. GDM was formed by adding GrB solutions to the DM prepared above, then incubating for $24 \mathrm{~h}$ at $4^{\circ} \mathrm{C}$. CC was selected as the model GrB protein for some in vitro evaluations. The preparation method of CC loaded mini micelles (CM) was the same as that of GM. The preparation method of CC/DTX co-loaded mini micelles (CDM) or Cy5.5-CC/C6 co-loaded mini micelles (CyC6M) was the same as that of GDM. CDM was mixed with mPEG- $b$-PELG at room temperature or below, and CDM-incorporated hydrogel (CDMH) was obtained by increasing the temperature to $37^{\circ}$ $\mathrm{C}$ in vitro or by injection into the body.

\section{Physicochemical Characterizations of Co-Loaded Mini Micelles-Incorporated Hydrogel}

Morphology of co-loaded mini micelles was observed by transmission electron microscopy (TEM) (HT7700, Japan). Particle size and zeta potential were determined using a Malvern Zetasizer Nano ZS instrument (ZS-90, Malvern, UK). Morphology of lyophilized CDMH was observed via scanning electron microscope (SEM) (JSM-6700F, Japan). And lyophilized Cy5.5-CC/C6 co-loaded mini micellesincorporated hydrogel was sliced and observed via confocal laser scanning microscopy (CLSM, LSM 780, Carl Zeiss, Germany). Rheological measurements of the hydrogel were performed by rheometer (MCR 302, Austria) using a parallel plate (plate diameter $=25 \mathrm{~mm}$, gap $=0.5 \mathrm{~mm}$ ) in oscillatory mode.

The concentration of DTX was determined by highperformance liquid chromatography (HPLC) (SHIMADZU LC-20AT, Japan). The concentration of CC was determined by Ultraviolet-visible spectroscopy (UV-vis). Drug loading efficiency (DL\%) and entrapment efficacy (EE\%) were calculated using the following equations:

$$
\mathrm{DL} \%=\frac{\mathrm{W}_{\mathrm{D}}}{\mathrm{W}_{\mathrm{T}}} * 100 \%
$$

where $\mathrm{W}_{\mathrm{D}}$ is the weight of drug analyzed, and $\mathrm{W}_{\mathrm{T}}$ is the total weight of drug and carrier added to the system.

$$
\mathrm{EE} \%=\frac{\mathrm{W}_{\mathrm{D}}}{\mathrm{W}_{\mathrm{TD}}} * 100 \%
$$

where $\mathrm{W}_{\mathrm{D}}$ is the weight of drug analyzed, and $\mathrm{W}_{\mathrm{TD}}$ is total weight of drug added to the system.

\section{In vitro Release of Cy5.5-CC and DTX}

Fluorescently labeled $\mathrm{CC}$ is often selected as the model $\mathrm{GrB}$ protein for in vitro evaluations. In vitro Cy5.5-CC and DTX release from mini micelles-incorporated hydrogel was performed using Transwell-24 (Corning). In brief, different formulations $(100 \mu \mathrm{L})$ were individually added to the 24well Transwell upper chamber and $1 \mathrm{~mL}$ of release medium (PBS at $\mathrm{pH} 7.4, \mathrm{pH} 6.5$ or $\mathrm{pH} 5.5$ ) was added to the lower chamber. The Transwell-24 plates were placed at $37^{\circ} \mathrm{C}$ with stirring at $100 \mathrm{rpm}$. At predetermined time intervals, the release medium was completely withdrawn, and replaced with fresh medium. The cumulative amounts of Cy5.5-CC and DTX in the collected samples were determined using a microplate reader and by HPLC, respectively. All measurements were performed in triplicate.

\section{In vivo Degradation and Biocompatibility of $\mathrm{mPEG}-b$-PELG}

In vivo degradation and biocompatibility of hydrogel materials mPEG- $b$-PELG were investigated using Kunming mice (weight: 18-22 g, age: 6-8 weeks). mPEG- $b$-PELG aqueous solution ( $0.1 \mathrm{~mL}, 6.0 \mathrm{wt} \%$ ) was subcutaneously injected into the back of mice. At selected time points, the mice were sacrificed. Then, the in situ hydrogel and skin tissues surrounding the hydrogel were surgically separated and observed. Hydrogel selected at different time points was weighed for in vivo characterization of hydrogel degradation, 
and the skin tissues were analyzed using hematoxylin and eosin staining for in vivo biocompatibility evaluation.

\section{Deep Tumor Penetration of Mini Micelles in vitro and in vivo}

The mini micelles were labeled by green fluorescent dye C6 to investigate the deep tumor penetration ability in the B16 tumor sphere. The preparation method of $\mathrm{C} 6$ loaded mini micelles $(\mathrm{C} 6 \mathrm{M})$ was the same as that of DM. C6M diluted by 1640 culture, were added to the well containing B16 tumor sphere at predetermined time points. After inoculation for $0.5 \mathrm{~h}, 2 \mathrm{~h}$ and $4 \mathrm{~h}$, the tumor spheres were washed 3 times with cold PBS and were imaged by a CLSM.

For deep tumor penetration in vivo evaluation, B16 tumor-bearing C57BL/6 mice were treated with $\mathrm{C} 6 \mathrm{M}$ incorporated hydrogel (C6MH) for $0.5 \mathrm{~h}, 4 \mathrm{~h}, 8 \mathrm{~h}, 12$ $\mathrm{h}$ and $24 \mathrm{~h}$. Then, the mice were sacrificed and the tumors were collected for paraffin embedding and tumor sections. After fixing the samples and staining the nuclei with DAPI, the sections were imaged by a CLSM.

\section{Lysosomal Escape of GrB}

To make the lysosomal escape visualized, Cy5.5-CC was selected as fluorescent labeled protein to replace $\mathrm{GrB}$. Briefly, B16 cells were cultured in glass bottom dish at a density of $1 \times 10^{5}$ cells per well. After overnight incubation, the cells were incubated with Lyso Tracker Red at $37^{\circ} \mathrm{C}$ for $2 \mathrm{~h}$ to label the lysosomes. After that, cells were treated with Cy5.5-CC loaded mini micelles (CyM) diluted by 1640 culture for $2 \mathrm{~h}$ or $4 \mathrm{~h}$ at $37{ }^{\circ} \mathrm{C}$ in an incubator. Then, the cells were washed 3 times with cold PBS and were imaged by a CLSM.

\section{In vitro and in vivo Co-Delivery Study of Co-Loaded Micelles-Incorporated Hydrogel}

Cy5.5-CC and $\mathrm{C} 6$ were selected as the fluorescent dyes to label the micelles for co-delivery study, in which Cy5.5-CC was in place of $\mathrm{GrB}$, and $\mathrm{C} 6$ was in place of DTX. CyC6M and $\mathrm{CyC} 6 \mathrm{M}$-incorporated hydrogel $(\mathrm{CyC} 6 \mathrm{MH})$ were prepared for in vitro and in vivo co-delivery study, respectively.

For in vitro co-delivery study, three groups were studied including (1) Mixture of free C6 and Cy5.5-CC; (2) Mixture of C6M and CyM; (3) CyC6M. B16 cells were cultured in glass-bottom dish at a density of $1 \times 10^{5}$ cells per well. After overnight incubation, the cells were incubated with different formulations diluted by 1640 culture for $0.5 \mathrm{~h}, 2 \mathrm{~h}$ or $4 \mathrm{~h}$ at $37^{\circ} \mathrm{C}$ in an incubator. Then, the cells were washed 3 times with cold PBS and were imaged by a CLSM.

For in vivo co-delivery study, three groups were studied including (1) Mixture of free C6 and Cy5.5-CC-incorporated hydrogel; (2) Mixture of C6M and CyM-incorporated hydrogel; (3) CyC6MH. B16 tumor-bearing C57BL/6 mice were treated with above formulations for $0.5 \mathrm{~h}, 4 \mathrm{~h}, 8 \mathrm{~h}, 12 \mathrm{~h}$ and $24 \mathrm{~h}$. Then, the mice were sacrificed and the tumors were collected for paraffin embedding and tumor sections. After fixing the samples and staining the nuclei with DAPI, the sections were imaged by a CLSM.

\section{In vitro Cytotoxicity of GDM}

In vitro cytotoxicity was evaluated on B16 cells via MTT assay. B16 cells were seeded in 96-well plates at a density of 5000 per well in $150 \mu \mathrm{L}$ of RPMI-1640 medium. After overnight incubation, the cells were treated with different formulations $(50 \mu \mathrm{L})$ at different concentrations and incubated for $48 \mathrm{hrs}$. Then $20 \mu \mathrm{L}$ of MTT $(5 \mathrm{mg} / \mathrm{mL})$ was added to each well for another $4 \mathrm{~h}$ incubation, dimethyl sulfoxide (DMSO) $(150 \mu \mathrm{L})$ was added to dissolve the formazan crystals, and absorbance value was determined at $570 \mathrm{~nm}$. Cell viability was calculated according to the following formula:

$$
\begin{aligned}
\text { Cell viability }(\%) & =\left(\mathrm{Abs}_{\text {sample }}-\mathrm{Abs}_{\text {blank }}\right) \\
& * 100 \% /\left(\mathrm{Abs}_{\mathrm{control}}-\mathrm{Abs}_{\text {blank }}\right)
\end{aligned}
$$

where Abs control, Abs sample, Abs blank represented the absorbance at $570 \mathrm{~nm}$ of the control, sample, blank, respectively.

Based on the cell viability values, concentration inhibiting half of the cells $\left(\mathrm{IC}_{50}\right)$ were calculated. Moreover, the synergistic effect between GrB and DTX was evaluated by the combination index $(\mathrm{CI})$ assay. $\mathrm{CI}$ values were calculated according to the following formula: ${ }^{38}$

$$
\mathrm{CI}=\mathrm{D}_{1} / \mathrm{D}_{\mathrm{m} 1}+\mathrm{D}_{2} / \mathrm{D}_{\mathrm{m} 2}
$$

where $\mathrm{D}_{1}$ or $\mathrm{D}_{2}$ was the concentration of drugs 1 or 2 when the combination of drug 1 and drug 2 produced $50 \%$ inhibition of cells. $\mathrm{D}_{\mathrm{m} 1}$ or $\mathrm{D}_{\mathrm{m} 2}$ was the concentration of the drug 1 or 2 when the drug produced $50 \%$ inhibition of cells via administered singly. The CI values lower than, equal to, and higher than 1 denote synergism, additivity and antagonism, respectively. 


\section{In vivo Antitumor Efficacy of GDMH}

B16 tumor-bearing female C57BL/6 mice model was established to demonstrate in vivo antitumor efficacy. And inhibition of tumor recurrence after surgery was studied simultaneously. $1 \times 10^{6}$ B16 cells were transplanted into the right flanks of mice. Five days after, all of the mice were randomly divided into two groups: Group A was divided into nine groups $(n=5){ }^{32,39}$ mice of Group B were anaesthetized, fixed to the board and visible tumors were resected by using sterile instruments, then were divided into nine groups $(n=5)$. Nine groups in Group A or B included: (1)Normal Solution; (2)Free DTX; (3)DTX loaded mini micelles (DM); (4) GrB loaded mini micelles (GM); (5)GrB/DTX co-loaded mini micelles (GDM); 6)Blank hydrogel; (7)DM-incorporated hydrogel (DMH); 8GM-incorporated hydrogel (GMH); (9)GDM-incorporated hydrogel (GDMH). One day after surgery, both Group A and B were treated with different formulations mentioned above (DTX: $2.5 \mathrm{mg} / \mathrm{kg}$; GrB: 0.95 $\mathrm{nmol} / \mathrm{kg}$ ) via local injection (peritumoral injection for A and surgical tumor bed injection for B). Ten days later, the second administration was conducted according to the first operation. Body weights and tumor volumes were recorded every other day. Tumor volumes were measured using a vernier caliper every other day by using the formula: $\left(\right.$ length $\times$ width $\left.^{2}\right) / 2$, where length was the longest dimension and width was the widest dimension. ${ }^{38}$ At the 14 th day after the first administration, the mice were sacrificed and anatomized. The excised tumors were weighed and analyzed via H\&E, Ki67, Caspase-3 and Tunel staining.

\section{In vivo Safety of GDMH}

The heart, liver, spleen, lung, and kidney of the mice in 2.13 were treated with formalin, embedded in paraffin and sectioned. Sections were stained with H\&E for histopathological examination to characterize the in vivo safety.

\section{Statistics Analysis}

All experimental data were presented as the mean \pm SD. Statistical differences were evaluated with $t$-Test (Excel 2016, Microsoft). Differences were considered to be significant when the $p$-value was less than 0.05 .

\section{Results}

\section{Characterization of PEG-b-PELG}

\section{Copolymer}

The structure and molecular weight were confirmed by ${ }^{1} \mathrm{H}$ NMR spectra on a Bruker AV 400 NMR spectrometer using Trifluoroacetic Acid-d as a solvent. Gel permeation chromatography (GPC) was performed using a series of liner Tskgel Super columns (AW3000 and AW5000) and Waters 515 HPLC pump with OPTILABDSP Interferometric Refractometer (Wyatt Technology) as the detector. The eluent was dimethylformamide containing $0.01 \mathrm{M} \mathrm{LiBr}$ at a flow rate of $1.0 \mathrm{~mL} / \mathrm{min}$ at $50^{\circ} \mathrm{C}$. Monodispersed polyethylene glycol (Waters) solutions were used to generate the calibration curve. Fourier Transform Infrared (FTIR) spectrum was recorded on a Bruker Vertex 70 Fourier Transform Infrared spectrometer using a pressed $\mathrm{KBr}$ disk method. For ${ }^{1}$ H NMR and FTIR of PEG-b-PELG (Figures S2-S3), all peaks have been well assigned. The molecular weight of the resulting copolymer, determined by GPC, was 4890 Da with a polydispersity index of 1.33 (Figure S4).

\section{Preparation and Characterization of Mini Micelles-Incorporated Hydrogel}

Mini micelles were prepared and characterized based on the different physicochemical factors of DTX and GrB. The PGA hydrophilic segment was designed for absorbing the positive GrB while the PLH pH-sensitive segment was designed hydrophobic DTX loading.

$\mathrm{CC}$ was replaced with $\mathrm{GrB}$ as the model protein for in vitro evaluations. As shown in Figure 2A-C and Table S1, CDM was near-

spherical and exhibited a small particle size $(26.90 \pm 0.99 \mathrm{~nm})$ with negatively charged surface $(-14.53 \pm 0.58 \mathrm{mV})$. The EE $\%$ was $70.84 \pm 1.53 \%$ for DTX and $90.95 \pm 8.37 \%$ for CC, respectively. The particle size and zeta potential of GDM or CyC6M were basically identical to those of CDM (Table S2).

In this study, changes in the size of blank micelles at different $\mathrm{pH}$ values indicate that micelle size was substantially unchanged at $\mathrm{pH}$ values of 7.4 and 6.5 , but rapidly increased when $\mathrm{pH}$ value was 5.5 (Figure S5). This is attributed to the $\mathrm{pH}$ sensitivity of PLH.

To demonstrate whether CC was loaded into the mini micelles, CM (the mass ratios of PLH-PGA:CC were from 20:1 to 400:1) were analyzed using sodium dodecyl sulfatepolyacrylamide gel electrophoresis. CC could be mostly loaded in the micelles after $24 \mathrm{~h}$ of incubation when the mass ratio was higher than 200:1 (Figure S6).

Due to the thermo-induced gelation behavior of mPEG$b$-PELG, the sol-gel phase transition was observed in CDMH when the temperature changed from room temperature to body temperature (Figure 2E). Mini micelles-incorporated hydrogel rheology experiments (storage modulus, loss 
A

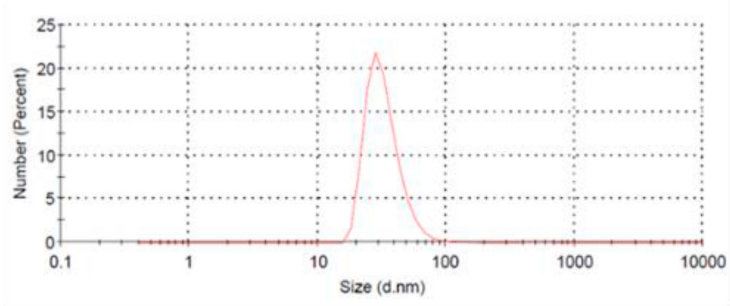

Size (d.nm)
B

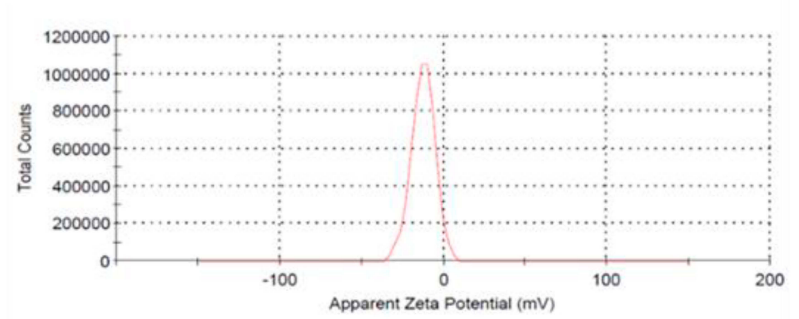

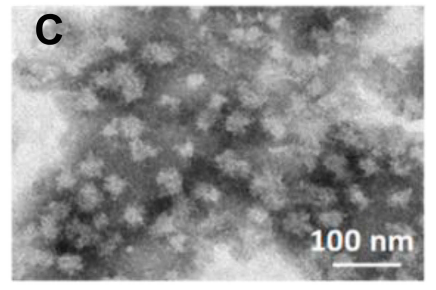

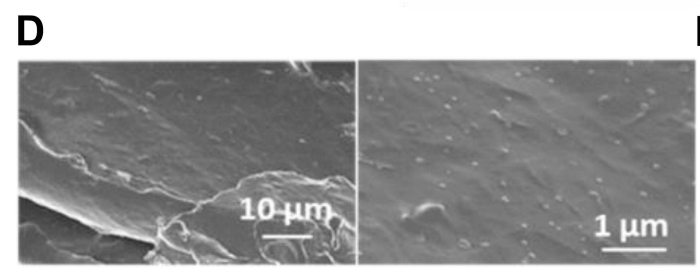

E

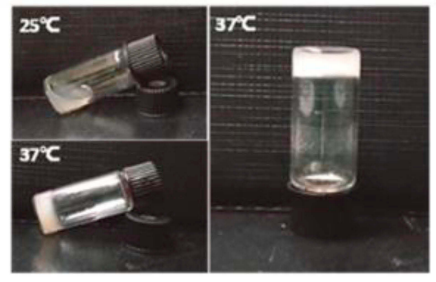

$\mathbf{F}$

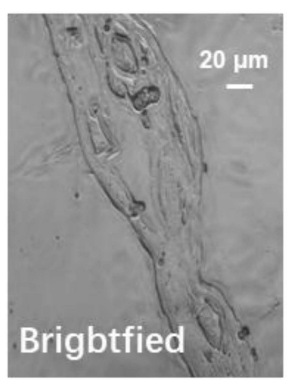

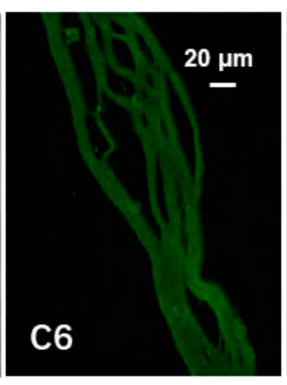
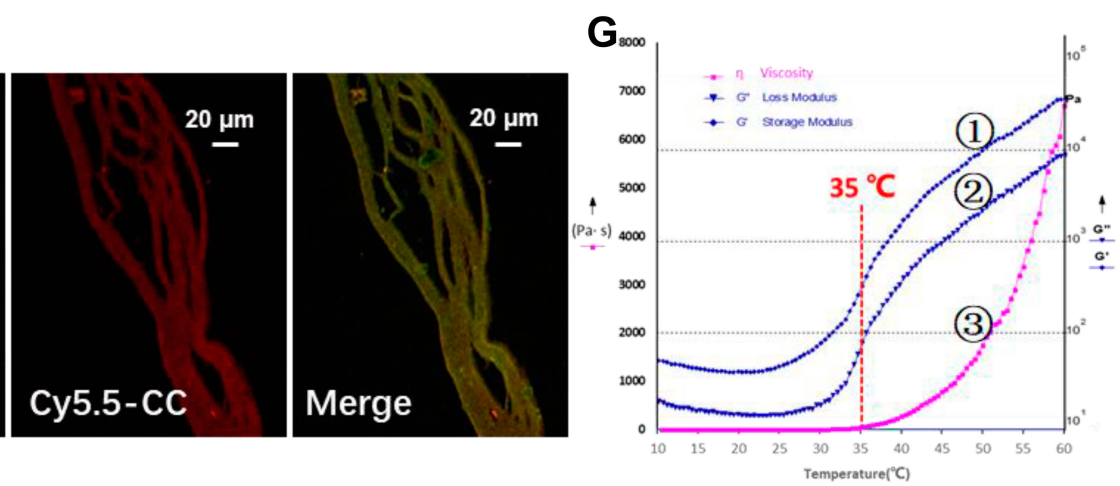

Figure 2 Characterizations of physical and chemical properties of CDM and CDMH. (A) Size distribution of CDM, for which the average size was $26.9 \pm 0.99 \mathrm{~nm}$. (B) Zeta potential distribution of CDM, for which the average zeta potential was $-14.53 \pm 0.58 \mathrm{mV}$. (C) Representative transmission electron microscope (TEM) images of CDM. (D) SEM images of lyophilized CDMH. (E) Photographs of the CDMH sol-to-gel transition with the increasing of temperature. (F) CLSM images of CyC6M-incorporated hydrogel, for which red, green and yellow color represent the fluorescence of Cy5.5-CC, C6 and the merged images, respectively. Based on the SEM images and CLSM, micelles could be homogeneously distributed in the hydrogel and could maintain the original structure for co-delivery. (G) Rheological properties of CDMH, for which G' (1), G” (2) and $\eta$ (3) represent the loss modulus, storage modulus and viscosity, for which $35^{\circ} \mathrm{C}$ could be the turning point of the CDMH system.

modulus, and viscosity) were performed and the results indicated that there was a catastrophe point at around $35^{\circ} \mathrm{C}$ after which the hydrogel system changed with increasing temperature (Figure 2G).

The hydrogel structure was studied using scanning electron microscopy (Figure 2D) and confocal laser scanning microscopy (Figure 2F), and CDM and CyC6M displayed uniform distributions in the lyophilized multilayer hydrogel structure.

\section{Drug Release in vitro and in vivo}

To evaluate the $\mathrm{pH}$-sensitive release behavior of both drugs, in vitro release was performed at different $\mathrm{pH}$ levels. Cy5.5-CC and DTX release profiles were quantified using a microplate reader and HPLC, respectively. Cy5.5-CC and DTX were rapidly released at $\mathrm{pH} 5.5$, but were slowly released at $\mathrm{pH} 7.4$ and $\mathrm{pH} 6.5$, which could be attributed to the $\mathrm{pH}$-sensitivity of the PLH. Moreover, at $\mathrm{pH} 6.5$, about $20 \%$ of DTX was released from the DMH or $\mathrm{CDMH}$, and $30 \%$ of Cy5.5-CC was released from the CyM-incorporated hydrogel or CyDM-incorporated hydrogel within 4 days (Figure 3A-D). The in situ formed mPEG- $b$-PELG hydrogel could be completely degraded in vivo within 21 days (Figure $3 \mathrm{E}$ and $\mathrm{F}$ ).

Furthermore, the deep tumor penetration ability of the mini micelles in vitro and in vivo was validated. The mini micelles showed a time-dependent deep tumor penetration in the 3D B16-tumor sphere and in B16 tumor-bearing female C57BL/6 mice (Figure 4A and B).

To demonstrate that GrB would not be degraded in the lysosome and achieve lysosomal escape with the help of the delivery carriers, Cy5.5-CC was selected as a model protein to visualize intracellular delivery. Most CyM Cy5.5-CC could escape from B16 cell lysosomes within $4 \mathrm{~h}$ (Figure 4C). 
A
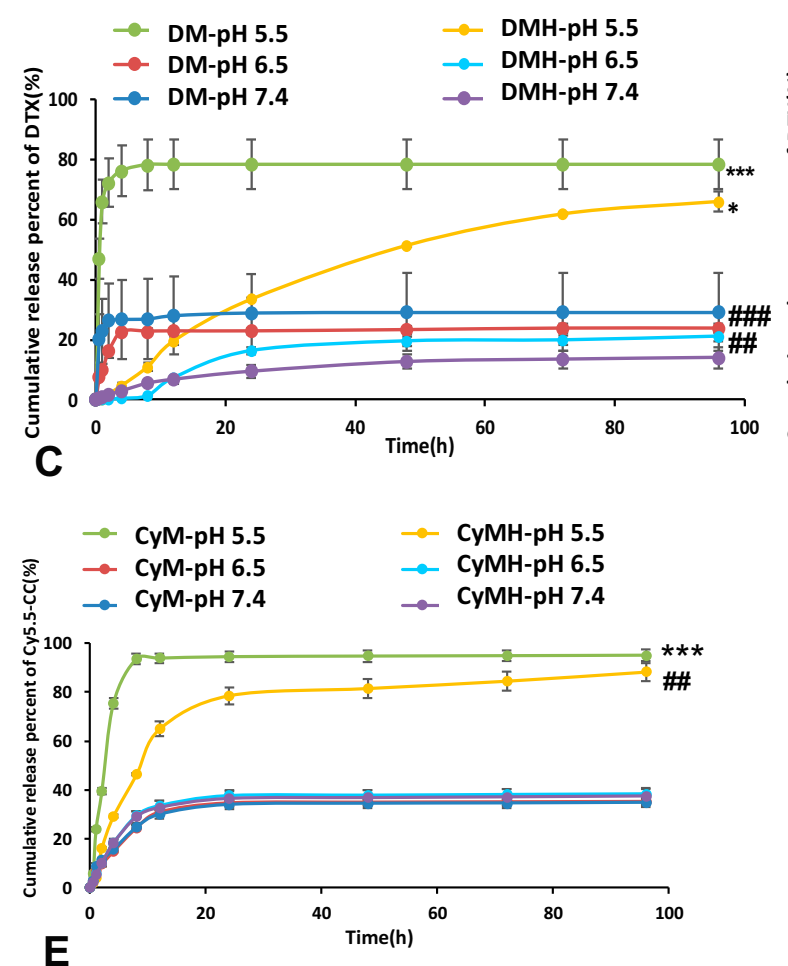

E

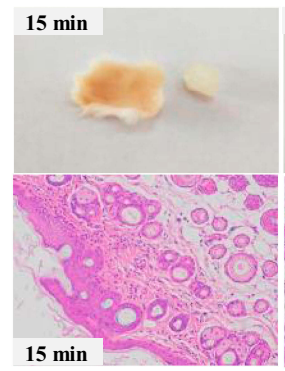

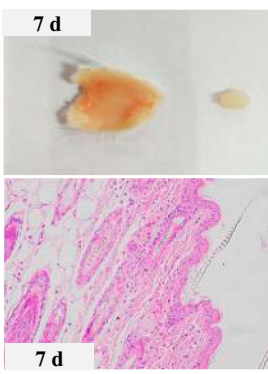

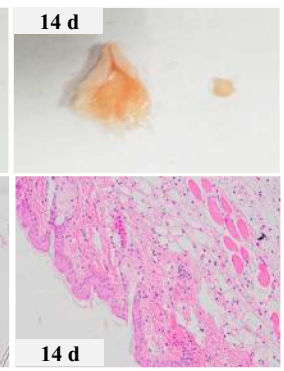

B
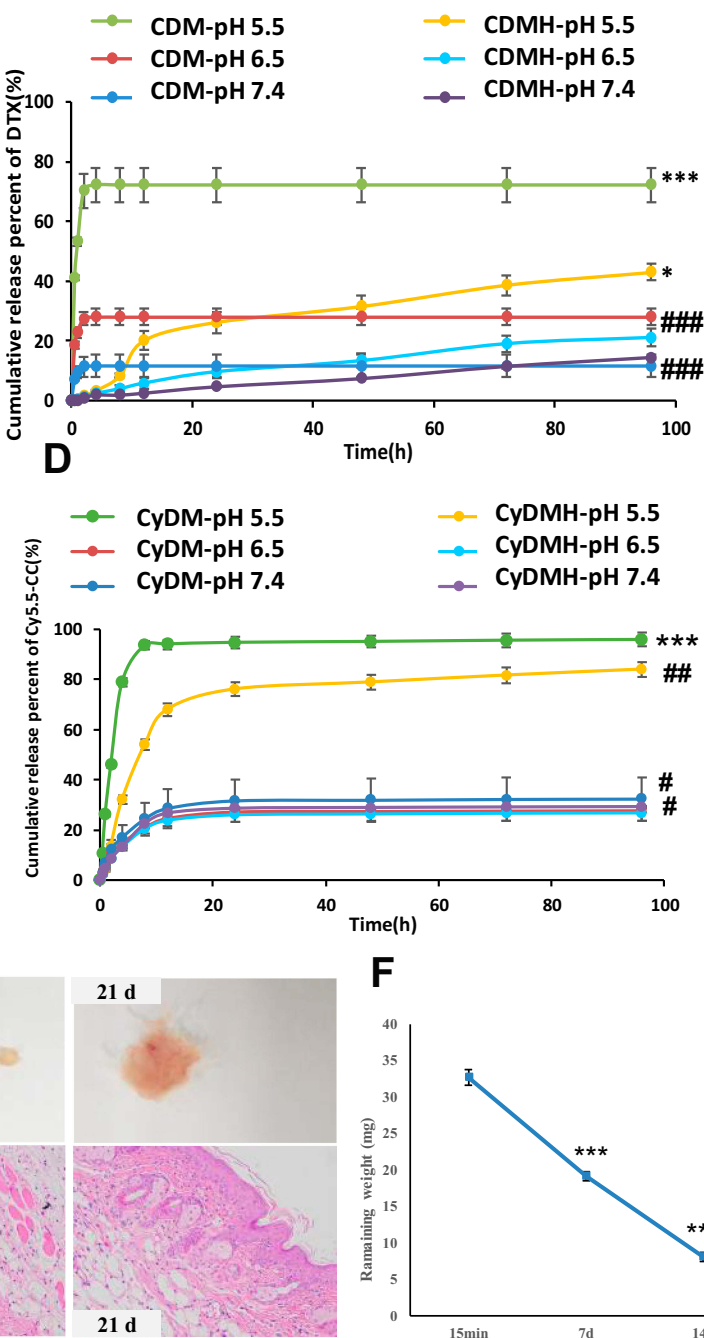

$\mathbf{F}$

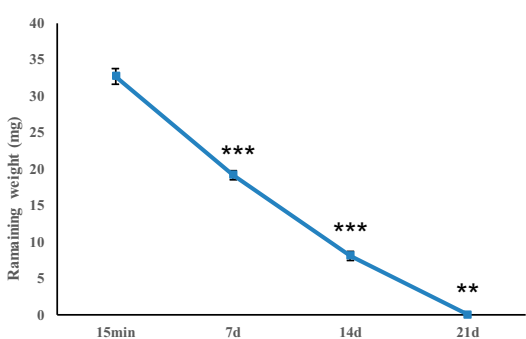

Figure 3 Characterizations of hydrogel for in vitro cumulative release profiles and in vivo degradation. (A) Cumulative release (\%) behavior of DTX from DM and DMH at different $\mathrm{pH}$ values. (B) Cumulative release (\%) behavior of DTX from CDM and CDMH at different $\mathrm{pH}$ values. (C) Cumulative release (\%) behavior of Cy5.5-CC from CyM and $\mathrm{CyMH}$ at different $\mathrm{pH}$ values. (D) Cumulative release (\%) behavior of Cy5.5-CC from CyDM and CyDMH at different $\mathrm{pH}$ values. (E) In situ hydrogel formation and in vivo hydrogel degradation. Histological images of the subcutaneous tissues surrounding the hydrogels (HE staining) (200x). (F) In vivo remaining weight for the in situforming mPEG-b-PELG (6.0 wt\%) hydrogel.

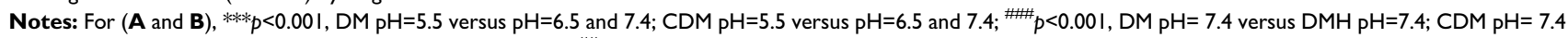

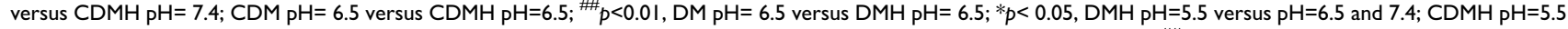
versus $\mathrm{pH}=6.5$ and 7.4. For $(\mathbf{C}$ and $\mathbf{D})$, ${ }^{* * *} \mathrm{p}<0.00 \mathrm{I}, \mathrm{CyM} \mathrm{pH}=5.5$ versus $\mathrm{pH}=6.5$ and $7.4 ; \mathrm{CyDM} \mathrm{pH}=5.5$ versus $\mathrm{pH}=6.5$ and 7.4 ; ${ }^{\#} p<0.0 \mathrm{I}, \mathrm{CyMH} p \mathrm{H}=5.5$ versus $\mathrm{pH}=6.5$ and

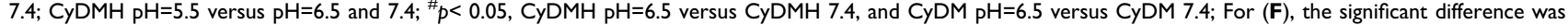
calculated by comparing the result of current weight with that of last weight, $* * * p<0.00$ I, current weight (7 d) versus last weight (I5 min), current weight (I4 $\mathrm{d}$ ) versus last weight (7 d); ${ }^{* *} p<0.01$, current weight $(2 \mathrm{l} d)$ versus last weight $(14 \mathrm{~d})$.

\section{Evaluation of Co-Delivery and Synergetic Therapeutic Efficacy}

Co-delivery of GrB and DTX into the same tumor cells is necessary to achieve synergistic therapeutic efficacy. CyC6M and $\mathrm{CyC} 6 \mathrm{MH}$ were prepared to assess the codelivery ability of the developed system in vitro and in vivo, respectively. B16 cells incubated with CyC6M had the strongest yellow fluorescence signal and the highest codelivery efficiency (95.14\%) at $4 \mathrm{~h}$, compared with cells that had a mixture of free $\mathrm{C} 6$ and $\mathrm{Cy} 5.5-\mathrm{CC}$, or a mixture of $\mathrm{C} 6 \mathrm{M}$ and $\mathrm{CyM}$ (Figure 5A and $\mathrm{B}$ ). Tumor sections from mice treated with $\mathrm{Cy} 5.5-\mathrm{CC}$ and $\mathrm{C} 6$ labeled hydrogel were observed using confocal laser scanning microscopy. The yellow fluorescence observed in the CyC6MH group was significantly stronger than that of the "mixture of free $\mathrm{C} 6$ and Cy5.5-CC-incorporated hydrogel" and "mixture of C6M and CyM-incorporated hydrogel" groups at $8 \mathrm{~h}$ and $12 \mathrm{~h}$ (Figure 5C). These data show that the co-delivery system 
A

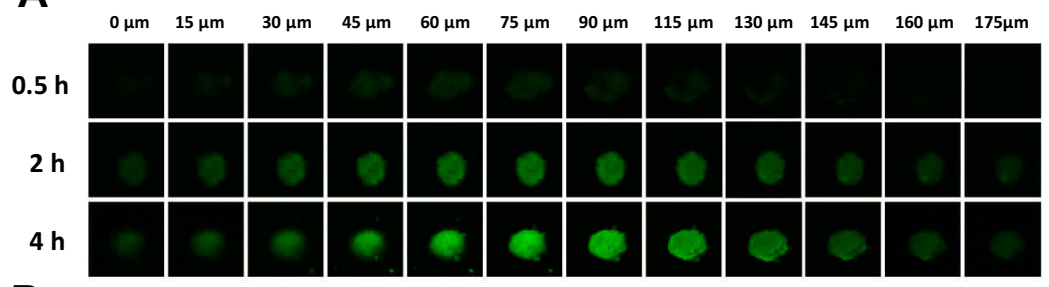

B

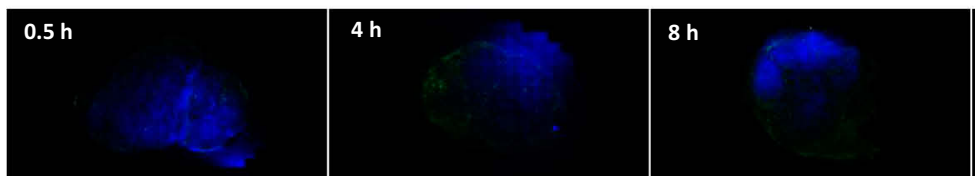

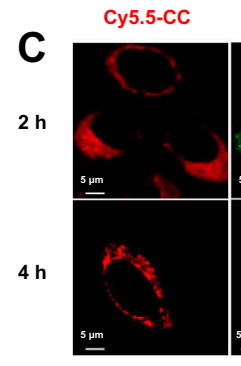

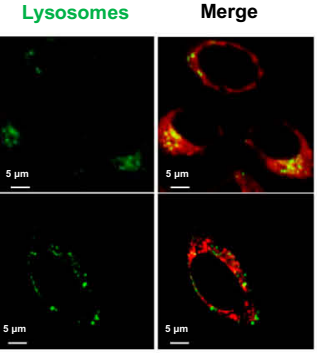

$12 \mathrm{~h}$
B

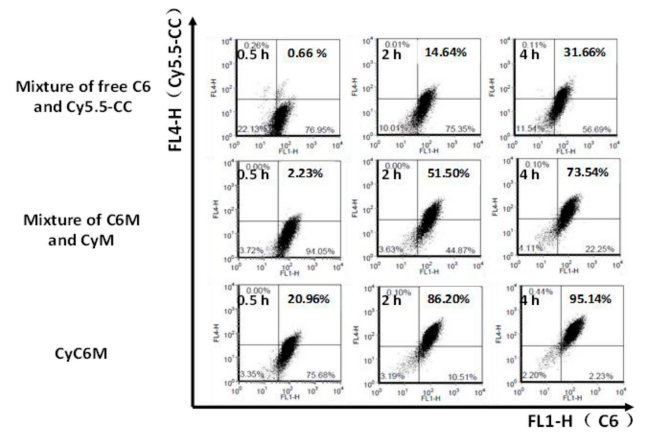

D
C

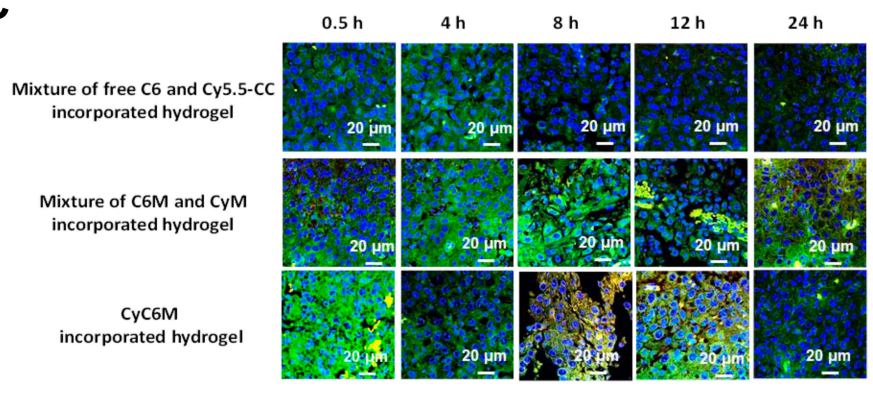

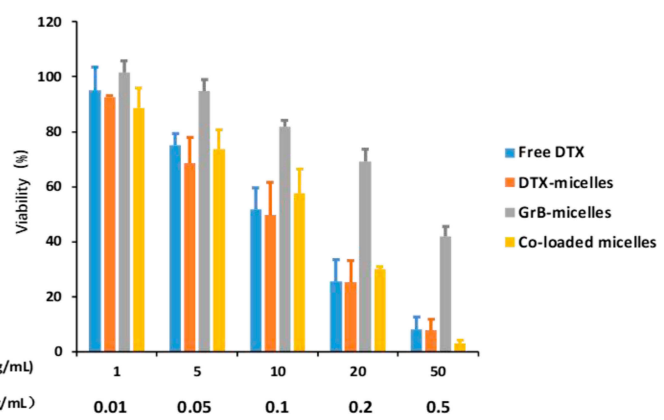

Figure 5 Characterizations of in vitro and in vivo co-delivery ability and in vitro cytotoxicity. For co-delivery study, Cy5.5-CC was selected for taking the place of GrB and C6 was selected for taking the place of DTX, respectively. For (A and B), first group was mixture of free C6 and Cy5.5-CC, the second group was mixture of C6M and $\mathrm{CyM}$, and the third group was CyC6M. For (C), first group was mixture of free $\mathrm{C} 6$ and $\mathrm{Cy} 5.5-\mathrm{CC}$-incorporated hydrogel, the second group was mixture of C6M and CyMincorporated hydrogel, and the third group was CyC6M-incorporated hydrogel. (A) CLSM images of cellular uptake at different time intervals on BI6 cells, in which red, green and yellow colors represent Cy5.5-CC, C6 and the merged images, respectively (magnification 63×, bar represents $20 \mu \mathrm{m}$, white arrow marked the two fluorescent drugs were delivered to the same cell). (B) Co-delivery efficiency evaluation of the mini micelles by flow cytometric analysis in BI6 cells. (C) CLSM images of tumor cyrosections after the mice were administrated with mixture of free drug (Cy5.5-CC and C6)-incorporated hydrogel (Lane I), mixture of Cy5.5-CC-micelles and C6-micellesincorporated hydrogel (Lane 2), and CyC6MH (Lane 3), for which blue, red, green and yellow color represent the fluorescence of DAPI, Cy5.5-CC, C6 and the merged images, respectively (magnification 63×, bar represents $20 \mu \mathrm{m}$ ). (D) In vitro cytotoxicity study of different formulations against BI6 cells. Data were given as mean \pm SD $(n=3)$. The concentrations of DTX and GrB were shown in x-axis.

designed in this study has superior potential to co-deliver different drugs into the same target cell/s.

The synergistic therapeutic efficacy of GrB and DTX was evaluated in B16 cells. Free GrB and PGA-PLH showed little cytotoxicity in B16 cells across a wide concentration range (Figure S7). Concentration-dependent cytotoxicity of free DTX, GM, DM, or GDM was observed (Figure 5D). The cytotoxicities of GrB and DTX at different mass ratios were 
characterized (Table S3). Maximum synergistic therapeutic efficacy was observed at 1:100 (CI values $=0.87)$. Thus, GDM (1:100) was applied in further experiments.

To investigate the synergistic therapeutic efficacy of the co-delivery system in vivo, the B16 tumor-bearing female C57BL/6 mice model was used, and post-surgical recurrence was observed (Figure 6A). After the mice were administered different formulations, the ninth group GDMH exhibited better antitumor efficacy than did GDM or DMH (Figure $6 \mathrm{~F}$ and $\mathrm{G})$. The excised tumors were photographed and weighed, and statistical analyses revealed that tumor weights and volumes were basically identical (Figure 6B-D). The body weights of the mice locally treated with GDMH did not significantly change during the treatment (Figure S8). Thus, GDMH treatment via peritumoral injection could effectively inhibit tumor growth and post-surgical recurrence (two-fifths of the mice in group (9) in Group B did not show tumor recurrence). The excised tumors were analyzed using hematoxylin and eosin, Ki67, Caspase-3, and Tunel staining. Compared with other groups, the group (9) both in Group $\mathrm{A}$ and $\mathrm{B}$ showed more tumor cell necrosis, up-regulated Caspase-3 levels, less tumor proliferation, and higher apoptosis levels (Figure 7). Histological analysis of organs harvested from the mice showed no obvious differences from the control (Figure 8).

\section{Discussion}

Combining chemotherapy and protein drugs are a widely employed strategy for tumor therapy. However, the application of chemo-protein combination therapy is limited by

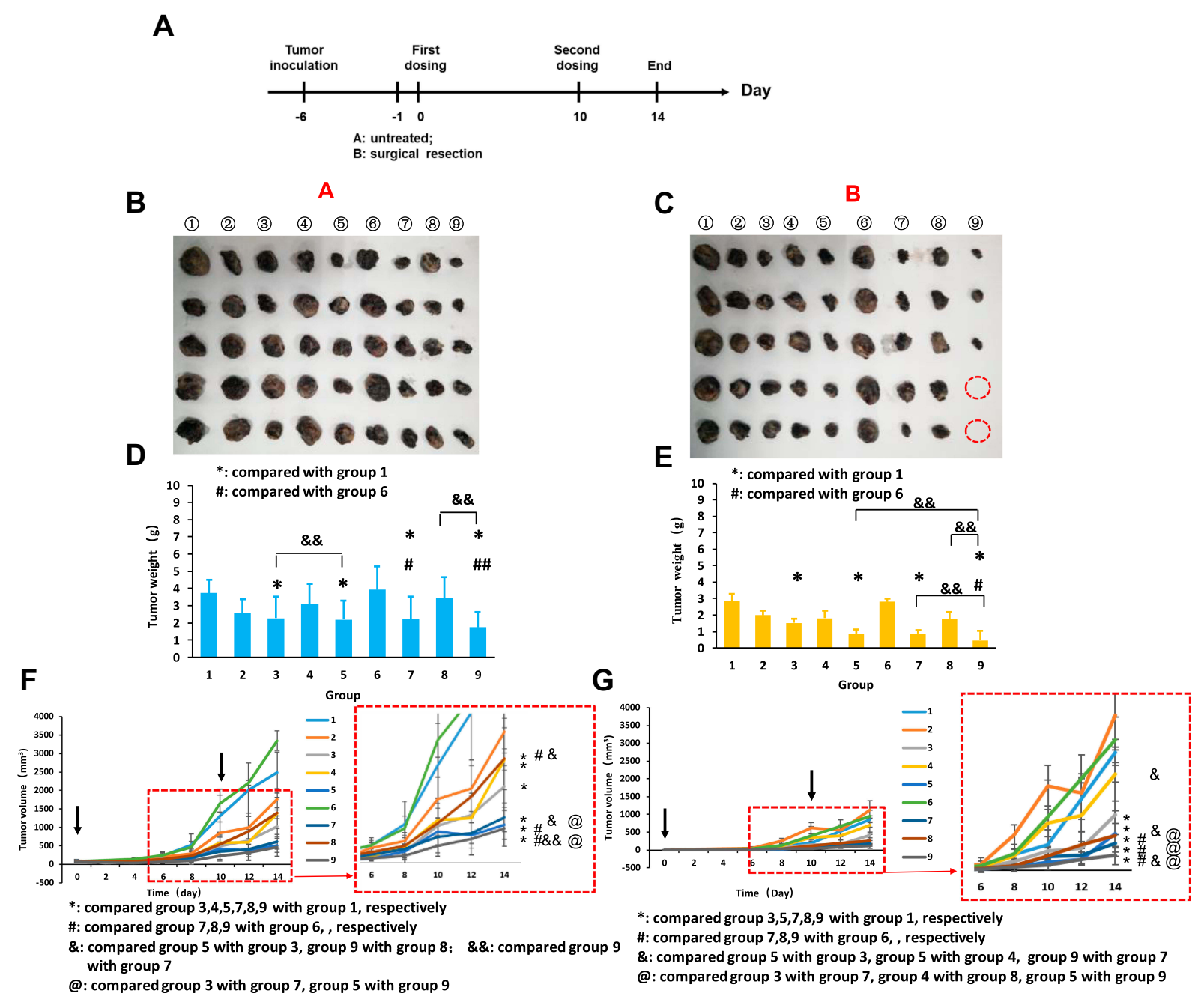

Figure 6 In vivo antitumor efficacy study. A: without surgery after tumor bearing; B: with surgery after tumor bearing. Nine groups in A or B including (1) Normal Solution; (2) Free DTX; (3) DM; (4) GM; (5) GDM; (6) Blank hydrogel; (7) DMH; (8) GMH; (9) GDMH. (A) Time points of in vivo anti-tumor activity study. (B) Photographs of tumors in A. (C) Photographs of tumors in B. (D) \&\&: $p<0.01$, group 5 versus group 3, group 9 versus group 8 ; \#\# : $<0.01$, group 9 versus group 6 ; \#: $p<0.05$, group 7 versus group 6 *: $p<0.05$, group 3 versus group I, group 5 versus group I, group 7 versus group I, group 9 versus group I. (E) \&\&: $p<0.0$ I, group 9 versus group 5 , group 9 versus group 7 , group 9 versus group 8; \#: $p<0.05$, group 9 versus group 6; *: $p<0.05$, group 3 versus group I, group 5 versus group I, group 7 versus group I, group 9 versus group I. (F) Tumor volume of Group A. (G) Tumor volume of Group B. Data were given as mean \pm SD $(n=5)$. 


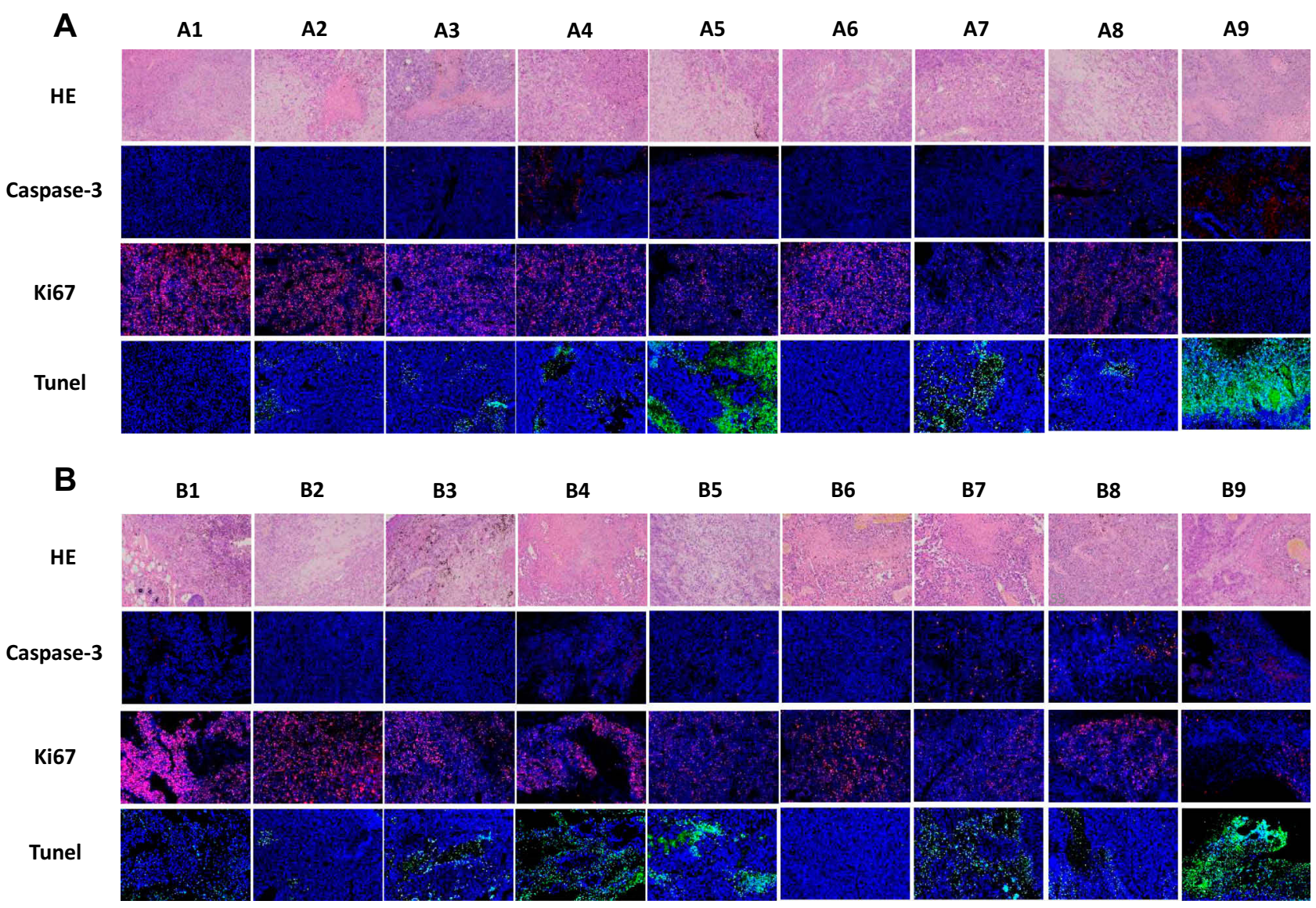

Figure 7 Representative microscopy images of H\&E (200x), Caspase-3 (200x), Ki67 (200x) and Tunel (200x) stained tumor histological sections after treatment with different formulations (A was for Group A, B was for Group B). (1) Normal Solution; (2) Free DTX; (3) DM; (4) GM; (5)GDM; (6) Blank hydrogel; (7) DMH; (8) GMH; (9) GDMH.

poor drug co-delivery efficacy, including low tumor accumulation, low tumor penetration, and unsatisfactory drug release due to the heterogenous nature of tumors and pharmacokinetic differences in drugs ${ }^{40}$. In this study, we successfully developed a tumor site-located, transformable hydrogel (GDMH) co-loaded with chemo-protein drugs. First, GrB/DTX was effectively co-loaded in $\mathrm{pH}$-sensitive micelles (GDM), and incorporated thermo-sensitive hydrogel (GDMH). GDM showed mini size and $\mathrm{pH}$-sensitive disassembly, which allowed for deep tumor penetration and rapid drug release (Figure 2A-D). GDMH underwent sol-gel phase transition when the temperature changed from room temperature to body temperature. The hydrogel viscosity showed a catastrophe point at around $35^{\circ} \mathrm{C}$, indicating that the hydrogel would turn from solution to gel at temperatures beyond $35^{\circ} \mathrm{C}$, and facilitate the encapsulation and controlled release of drugs (Figure 2E-G).

After accumulation at the tumor site, drug-delivery carriers need to be transported into deep tumor tissue. However, tumor tissues are dense, with high pressure and lack of blood vessels. This makes it difficult for carriers to deeply penetrate into the tumor site and when administered intravenously they only damage the cells around the blood vessels, limiting their antitumor effect. Small-sized nanoparticles (less than $50 \mathrm{~nm}$ ) exhibited significant benefits for deep tumor penetration 35 . We confirmed that the hydrogel could be completely degraded within 21 days (Figure 3E and F), which is beneficial for the release of mini micelles. Furthermore, we found that the released mini micelles could deeply penetrate into the tumor site (Figure 4A and B).

The synergistic therapeutic effects of combination therapy drugs that act on tumor cells are dependent on their ability to enter the same tumor cell while maintaining their original structure and rapidly releasing into the tumor cell38. We confirmed that the transformable co-loaded hydrogel showed the highest co-delivery efficiency of the groups examined in vitro and in vivo. Once delivered into the tumor, the drugs should be effectively released to exert 

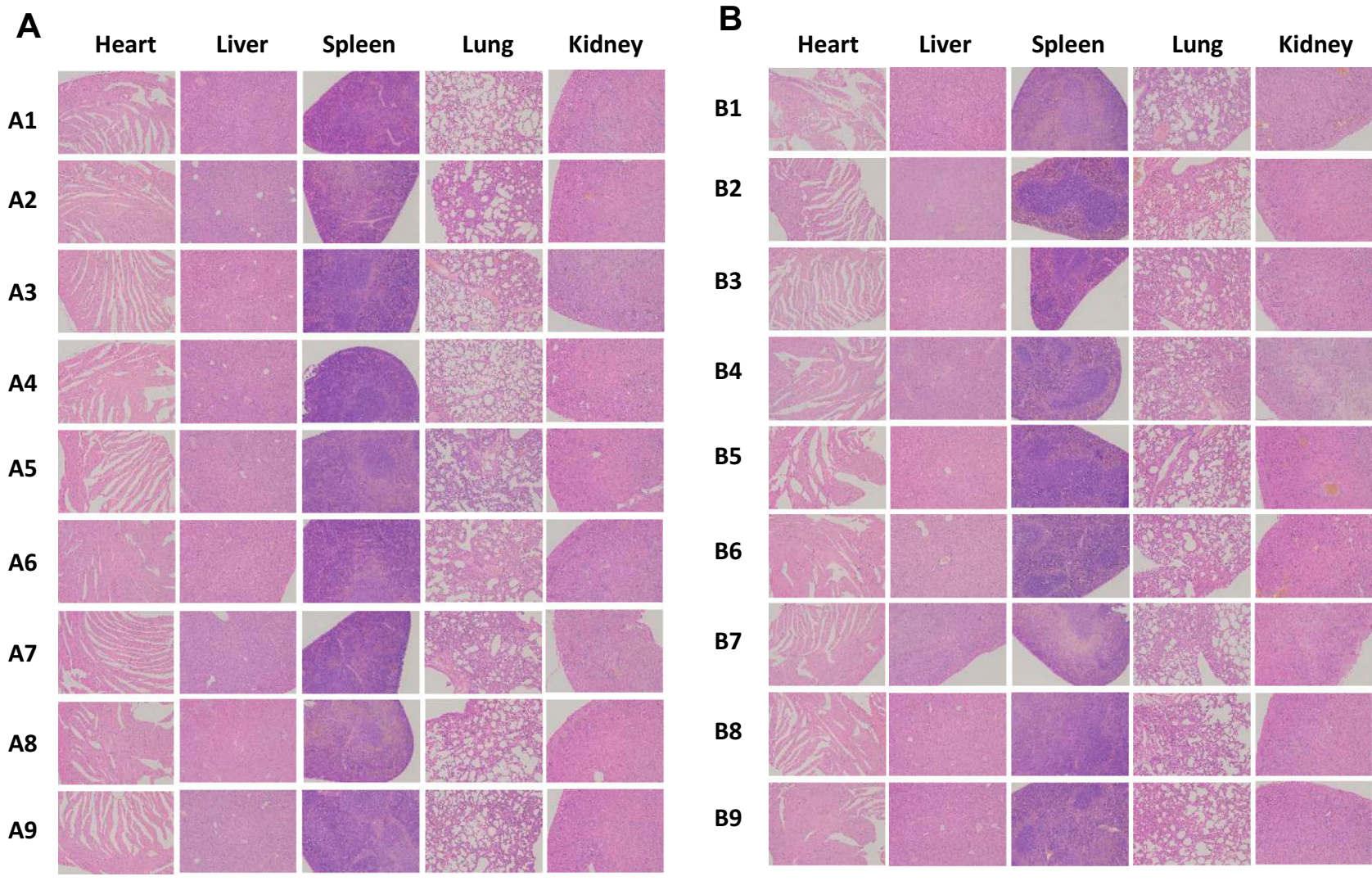

Figure 8 In vivo security evaluation. Representative microscopy images of H\&E stained histological sections on mice after treatment with different formulations (I00x) (A was for Group A, B was for Group B). (1) Normal Solution; (2) Free DTX; (3) DM; (4) GM; (5) GDM; (6) Blank hydrogel; (7) DMH; (8) GMH; (9) GDMH.

their antitumor efficacy. Our results show that the coloaded transformable hydrogels developed in this study show rapid drug release under $\mathrm{pH} 5.5$, and slower drug release at $\mathrm{pH} 7.4$ and $\mathrm{pH}$ 6.5. This could be attributed to the pH-sensitivity of the PLH core (Figure 3A-D). Furthermore, we observed that the co-loaded mini micelles escaped from the lysosome, confirming that mini micelles can protect $\mathrm{GrB}$ from lysosome-mediated degradation and release GrB into the cytoplasm (Figure 4C).

To investigate the in vivo synergistic therapeutics of GDMH, the B16 tumor-bearing female C57BL/6 mice model was used, and post-surgical recurrence was observed. The combination of GrB and DTX in transformable GDMH exhibited the greatest tumor inhibition, and prevented postoperative tumor recurrence (Figures $6 \mathrm{~A}-\mathrm{G}$ and 7). The observed inhibition of tumor growth might result from: (i) effective tumor accumulation of transformable hydrogel; (ii) deep tumor penetration of the co-loaded $\mathrm{pH}$-sensitive mini micelles; or (iii) co-loaded $\mathrm{pH}$-sensitive mini micelles internalization into the same tumor cell, then disassembly under the acid environment and rapid drug release to synergistically inhibit tumor cell growth. Histology analysis of organs exhibited good biocompatibility and safety (Figure 8).

We report, for the first time, a transformable delivery system to co-load GrB/DTX. The transformable hydrogel could achieve sequentially responsive delivery by three steps: (i) gelatinization of GDMH at body temperature to colocate two drugs at the tumor site; (ii) GDMH proteinase degradation and mini micelle release to achieve deep tumor penetration; and (iii) mini micelles disassembly and escape from lysosomes to release GrB and DTX. The smart-designed transformable GDMH will pave a new way for the design of delivery systems to co-deliver drugs with different pharmacokinetics, facilitate tumor accumulation, ensure deep tumor penetration and rapid release of drugs, and achieve synergistic chemo-protein therapy.

\section{Conclusions}

In summary, GDMH, a structure-transformable, thermo-pH responsive co-delivery system that delivers co-loaded chemo-protein drugs to tumor sites, transports drugs deep into the tumor tissue, and rapidly releases drug in the tumor 
cells was successfully developed. GDMH is composed of hydrogel material (mPEG- $b$-PELG) and mini micelles. mPEG- $b$-PELG can be gelatinized at body temperature and is gradually degraded by proteinase in vivo, allowing for the exposure of mini-sized GDM with deep tumor-penetrating ability. The exposed mini GDM, containing acid-sensitive PGA-PLH, has properties of $\mathrm{pH}$-sensitive drug release and lysosomal escape due to the PLH side-chain imidazole groups. GrB and DTX could be synchronously internalized into the same tumor cells and had synergistic therapeutic efficacy at the mass ratio of 1:100. More importantly, the combination of GrB and DTX exhibited high tumor inhibition in both the subcutaneous tumor model and the postsurgical recurrence model at a low dose. Additionally, GDMH showed good biocompatibility and safety. These results show that the clinical translation of combining chemotherapy and protein drugs has great therapeutic potential. The smart-designed transformable GDMH will pave a new way for designing delivery systems for the co-delivery of drugs with different pharmacokinetics, and facilitate tumor accumulation, deep tumor penetration, and rapid drug release to achieve synergistic chemo-protein therapy.

\section{Funding}

This work was supported by the National Natural Science Foundation of China (grant numbers: 81773652, 81974498, and 51622307).

\section{Disclosure}

The authors declare that there are no conflicts of interest.

\section{References}

1. Enriquez-Navas PM, Wojtkowiak JW, Gatenby RA. Application of evolutionary principles to cancer therapy. Cancer Res. 2015;75 (22):4675-4680. doi:10.1158/0008-5472.CAN-15-1337

2. He CL, Tang ZH, Tian HY, Chen XS. Co-delivery of chemotherapeutics and proteins for synergistic therapy. Adv Drug Deliver Rev. 2016;98:64-76. doi:10.1016/j.addr.2015.10.021

3. Hiller JG, Perry NJ, Poulogiannis G, Riedel B, Sloan EK. Perioperative events influence cancer recurrence risk after surgery. Nat Rev Clin Oncol. 2018;15(4):205-218. doi:10.1038/nrclinonc. 2017.194

4. Gao WP, Sun MM. Drug nanorods are potential new nanocarriers for intracellular protein delivery. Theranostics. 2018;8(14):3872-3873. doi: $10.7150 /$ thno. 27815

5. Schoonen L, van Hest JCM. Functionalization of protein-based nanocages for drug delivery applications. Nanoscale. 2014;6(13):7124-7141. doi:10.1039/C4NR00915K

6. Lu L, Zou Y, Yang WJ, et al. Anisamide-decorated pH-sensitive degradable chimaeric polymersomes mediate potent and targeted protein delivery to lung cancer cells. Biomacromolecules. 2015;16 (6):1726-1735. doi:10.1021/acs.biomac.5b00193
7. Rousalova I, Krepela E. Granzyme B-induced apoptosis in cancer cells and its regulation (Review). Int J Oncol. 2010;37(6):1361-1378. doi:10.3892/ijo_00000788

8. Shi L, Keefe D, Durand E, Feng HP, Zhang D, Lieberman J. Granzyme B binds to target cells mostly by charge and must be added at the same time as perforin to trigger apoptosis. $J$ Immunol. 2005;174(9):5456-5461. doi:10.4049/jimmunol.174.9.5456

9. Oberoi P, Jabulowsky RA, Bahr-Mahmud H, Wels WS. EGFRtargeted granzyme B expressed in NK cells enhances natural cytotoxicity and mediates specific killing of tumor cells. PLoS One. 2013;8:4. doi:10.1371/journal.pone.0061267

10. Yang WJ, Wei YH, Yang L, et al. Granzyme B-loaded, cell-selective penetrating and reduction-responsive polymersomes effectively inhibit progression of orthotopic human lung tumor in vivo. $J$ Control Release. 2018;290:141-149. doi:10.1016/j.jconrel.2018.10.013

11. Amoury M, Kolberg K, Pham AT, et al. Granzyme B-based cytolytic fusion protein targeting EpCAM specifically kills triple negative breast cancer cells in vitro and inhibits tumor growth in a subcutaneous mouse tumor model. Cancer Lett. 2016;372 (2):201-209. doi:10.1016/j.canlet.2016.01.027

12. de Saint Basile G, Menasche G, Fischer A. Molecular mechanisms of biogenesis and exocytosis of cytotoxic granules. Nat Rev Immunol. 2010;10(8):568-579. doi:10.1038/nri2803

13. Hlongwane $P$, Mungra N, Madheswaran S, Akinrinmade OA, Chetty S, Barth S. Human granzyme B based targeted cytolytic fusion proteins. Biomedicines. 2018;6(2):72. doi:10.3390/biomedicines60 20072

14. Lv XZ, Zheng MY, Lin ZQ, Zhao M, Wang H, Zeng WS. Granzyme B-truncated VEGF fusion protein represses angiogenesis and tumor growth of OSCC. Oral Dis. 2016;22(7):688-696. doi:10.1111/ odi. 12522

15. Long DW, Johnson NR, Jeffries EM, Hara H, Wang YD. Controlled delivery of platelet-derived proteins enhances porcine wound healing. J Control Release. 2017;253:73-81. doi:10.1016/j.jconrel.2017.03. 021

16. Pachioni-Vasconcelos JD, Lopes AM, Apolinario AC, et al. Nanostructures for protein drug delivery. Biomater Sci. 2016;4 (2):205-218. doi:10.1039/C5BM00360A

17. Jang B, Kwon H, Katila P, Lee SJ, Lee H. Dual delivery of biological therapeutics for multimodal and synergistic cancer therapies. $A d v$ Drug Deliver Rev. 2016;98:113-133. doi:10.1016/j.addr.2015.10.023

18. Lee A, Djamgoz MBA. Triple negative breast cancer: emerging therapeutic modalities and novel combination therapies. Cancer Treat Rev. 2018;62:110-122. doi:10.1016/j.ctrv.2017.11.003

19. Patel SA, Minn AJ. Combination cancer therapy with immune checkpoint blockade: mechanisms and strategies. Immunity. 2018;48 (3):417-433. doi:10.1016/j.immuni.2018.03.007

20. Rosenblum D, Joshi N, Tao W, Karp JM, Peer D. Progress and challenges towards targeted delivery of cancer therapeutics. Nat Commun. 2018;9. doi:10.1038/s41467-018-03705-y

21. Zhang Y, Tan XY, Ren TY, Jia C, Yang ZX, Sun H. Folate-modified carboxymethyl-chitosan/polyethylenimine/bovine serum albumin based complexes for tumor site-specific drug delivery. Carbohyd Polym. 2018;198:76-85. doi:10.1016/j.carbpol.2018.06.055

22. Wu TT, Qiao Q, Qin XY, Zhang D, Zhang ZP. Immunostimulatory cytokine and doxorubicin co-loaded nanovesicles for cancer immunochemotherapy. Nanomed-Nanotechnol. 2019;18:66-77. doi:10.1016/j.nano.2019.02.008

23. Chen J, Ouyang J, Chen QJ, et al. EGFR and CD44 dual-targeted multifunctional hyaluronic acid nanogels boost protein delivery to ovarian and breast cancers in vitro and in vivo. ACS Appl Mater Inter. 2017;9(28):24140-24147. doi:10.1021/acsami.7b06879

24. Chen J, Zou Y, Deng C, Meng FH, Zhang J, Zhong ZY. Multifunctional click hyaluronic acid nanogels for targeted protein delivery and effective cancer treatment in vivo. Chem Mater. 2016;28 (23):8792-8799. doi:10.1021/acs.chemmater.6b04404 
25. Vermonden T, Censi R, Hennink WE. Hydrogels for protein delivery. Chem Rev. 2012;112(5):2853-2888. doi:10.1021/cr200157d

26. Ding D, Zhu ZS, Li RT, et al. Nanospheres-incorporated implantable hydrogel as a trans-tissue drug delivery system. ACS Nano. 2011;5 (4):2520-2534. doi:10.1021/nn102138u

27. Huang PS, Song HJ, Zhang YM, et al. Bridging the gap between macroscale drug delivery systems and nanomedicines: a nanoparticle-assembled thermosensitive hydrogel for peritumoral chemotherapy. ACS Appl Mater Inter. 2016;8(43):29323-29333. doi:10.1021/acsami.6b10416

28. Ruan HT, Hu QY, Wen D, et al. A dual-bioresponsive drug-delivery depot for combination of epigenetic modulation and immune checkpoint blockade. Adv Mater. 2019;31(17). doi:10.1002/adma.2018 02348 .

29. Zheng Y, Wang W, Zhao J, et al. Preparation of injectable temperature-sensitive chitosan-based hydrogel for combined hyperthermia and chemotherapy of colon cancer. Carbohydr Polym. 2019;222:115039. doi:10.1016/j.carbpol.2019.115039

30. Liu YY, Xi YX, Zhao JL, et al. Preparation of therapeutic-laden konjac hydrogel for tumor combination therapy. Chem Eng J. 2019;375:122048. doi:10.1016/j.cej.2019.122048

31. Bastiancich C, Danhier P, Preat V, Danhier F. Anticancer drug-loaded hydrogels as drug delivery systems for the local treatment of glioblastoma. J Control Release. 2016;243:29-42. doi:10.1016/j. jconrel.2016.09.034

32. Wang C, Wang JQ, Zhang XD, et al. In situ formed reactive oxygen species-responsive scaffold with gemcitabine and checkpoint inhibitor for combination therapy. Sci Transl Med. 2018;10(429):eaan3682. doi:10.1126/scitranslmed.aan3682

33. Wu XL, He CL, Wu YD, Chen XS, Cheng JJ. Nanogel-incorporated physical and chemical hybrid gels for highly effective chemo-protein combination therapy. Adv Funct Mater. 2015;25(43):6744-6755. doi:10.1002/adfm.201502742
34. Yu ML, Zhang CX, Tang ZH, Tang X, Xu H. Intratumoral injection of gels containing losartan microspheres and (PLG-g-mPEG)cisplatin nanoparticles improves drug penetration, retention and anti-tumor activity. Cancer Lett. 2019;442:396-408. doi:10.1016/j. canlet.2018.11.011

35. Hu C, Cun XL, Ruan SB, et al. Enzyme-triggered size shrink and laser-enhanced NO release nanoparticles for deep tumor penetration and combination therapy. Biomaterials. 2018;168:64-75. doi:10.1016/ j.biomaterials.2018.03.046

36. Son S, Deepagan VG, Shin S, et al. Ultrasmall gold nanosatellite-bearing transformable hybrid nanoparticles for deep tumor penetration. Acta Biomater. 2018;79:294-305. doi:10.1016/j. actbio.2018.08.019

37. Wu X, Wu Y, Ye H, Yu S, He C, Chen X. Interleukin-15 and cisplatin co-encapsulated thermosensitive polypeptide hydrogels for combined immuno-chemotherapy. $J$ Control Release. 2017;255:81-93. doi:10.1016/j.jconrel.2017.04.011

38. Zhang B, Wang TQ, Yang SM, et al. Development and evaluation of oxaliplatin and irinotecan co-loaded liposomes for enhanced colorectal cancer therapy. $J$ Control Release. 2016;238:10-21. doi:10.1016/j.jconrel.2016.07.022

39. Chen Q, Wang C, Zhang XD, et al. In situ sprayed bioresponsive immunotherapeutic gel for post-surgical cancer treatment. Nat Nanotechnol. 2019;14(1):89-+. doi:10.1038/s41565-018-0319-4

40. Song Q, Yin Y, Shang L, et al. Tumor microenvironment responsive nanogel for the combinatorial antitumor effect of chemotherapy and immunotherapy. Nano Lett. 2017;17(10):6366-6375. doi:10.1021/ acs.nanolett. $7 \mathrm{~b} 03186$
International Journal of Nanomedicine

\section{Publish your work in this journal}

The International Journal of Nanomedicine is an international, peerreviewed journal focusing on the application of nanotechnology in diagnostics, therapeutics, and drug delivery systems throughout the biomedical field. This journal is indexed on PubMed Central, MedLine, CAS, SciSearch ${ }^{\mathbb{}}$, Current Contents ${ }^{\mathbb{R}} /$ Clinical Medicine,

\section{Dovepress}

Journal Citation Reports/Science Edition, EMBase, Scopus and the Elsevier Bibliographic databases. The manuscript management system is completely online and includes a very quick and fair peer-review system, which is all easy to use. Visit http://www.dovepress.com/ testimonials.php to read real quotes from published authors. 